\title{
Deformation Research of Titanium Alloy Diaphragm Disc With Different Cutting Edge Radius
}

\author{
Aiqin Lin \\ Department of Information Engineering, Liaoning Economic Management Cadre Institute, \\ Shenyang, 110122, China
}

Email: laq1206@163.com

\begin{abstract}
Keywords:titanium alloy diaphragm disc; cutting edge type; precision cutting; theory model Abstract.In different parts of cutting force, it is researched cutting force on machining titanium alloy by the way of theory model and finite element analytical. Established cutting force model of titanium alloy precision machining. Analysis distribution of cutting tool edge stress field caused by different cutter blade type and provides the theory basis for choice of disc cutter blade type in precision cutting of titanium alloy membrane, further provides the theoretical foundation to improve titanium alloy membrane plate yield.
\end{abstract}

\section{Introduction}

Titanium alloy diaphragm disc is a typical thin-walled parts in aircraft engine. There are light weight, compact structure, material saving. It has been widely used in high power, high speed transmission.In cutting force, cutting heat, residual stress and other factors, the disc surface of titanium alloy membranes is seriouslychanged. They have severely affected production efficiency and yield. With the development of aerospace enterprise, the requirement of workpiece machining accuracy will become highly. Suppress the workpiece deformation in the process get the attention of people.

\section{The structure characteristics of titanium alloy diaphragm disc}

Surface of titanium alloy diaphragm disc is complex, the two sides for the quadratic equation. It is poor rigidity. The thinnest place of surface is only $0.446 \mathrm{~mm}$,High machining precision requirement,Membrane on the surface of the disc require $0.025 \mathrm{~mm}$. Its surface roughness require $0.2 \mu \mathrm{m}$. The structure diagram of titanium alloy diaphragm disc is shown in Figure 1.

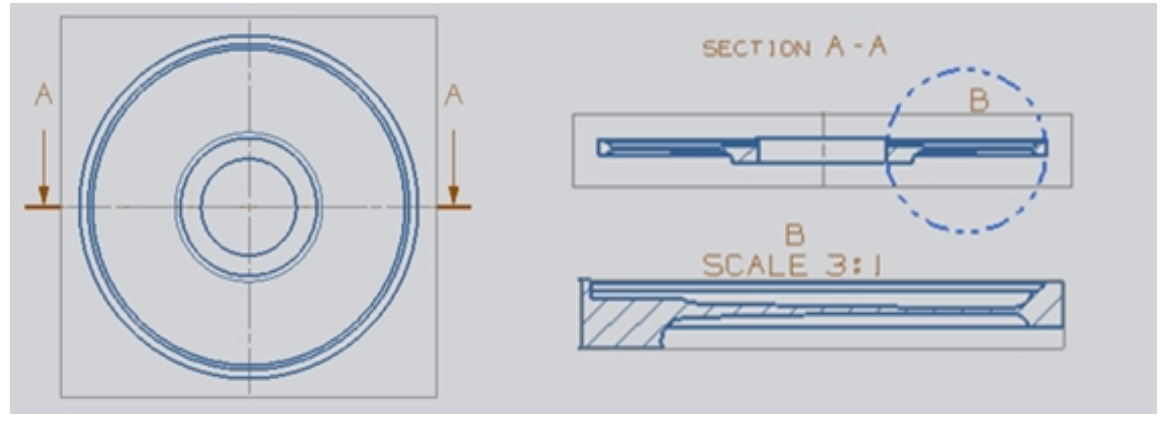

Fig. 1 The structure of titanium alloy diaphragm disc

\section{Cutting mechanics model of blade obtuse and chamfering edge}

Due to the cutter blade obtuse, removed workpiece material won't like a sharp blade all through the rake face and chip outflow,but exists a 0 points of material flow velocity in the blade, known as split points.Under the condition of the same size, with the different diversion point position of cutting thickness, affecting the cutting force of numerical values.Cutting model of considering cutting tool edge blunt is shown in Figure 2.As you can see from figure 2, the introduction of the 
blade obtuse force, classic cutting force attached blade blunt force influence. The influence of the workpiece material flow is analyzed in diversion point and more close actual cutting process .It can more truly reflect the actual machining process.

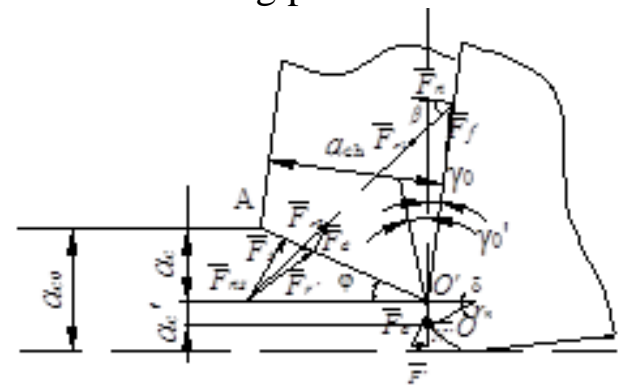

Fig.2 Two-dimensional orthogonal cutting model incorporating tool round edge

Among them, $\delta$ is round central angle of cutting edge blunt round. $\gamma_{0}^{\prime}$ is the actual rake angle of cutting edge area. $r_{n}$ is cutting edge blunt round radius, $a_{c 0}$ is cutting thickness. $a_{c}^{\prime}$ is cutting thickness of vertically cutting edge blunt round distribution points to the rake face. $A$ is corresponding area of $O O^{\prime}$ arc length. At this point, the stress force of blade obtuse area is due to the cutting edge blunt round crushing obtuse materials. When cutter blade type is chamfer edge,chamfering edge cutting force $\breve{F}_{e}$ in chamfering edge can be decomposed into parallel cutting force $\breve{F}^{\prime}$ and cutting force $\breve{F}^{\prime}$ of horizontal workpiece surface. Chamfering edge cutting mechanics model is shown in Fig 3.

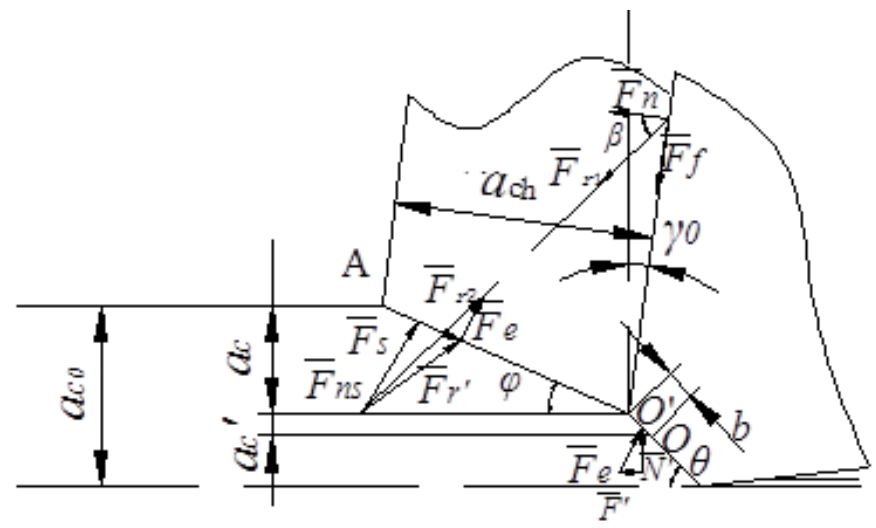

Fig.3 Two-dimensional orthogonal cutting model incorporating chamfering edge

Based on the above analysis, we can know that cutting force attached force from cutting edge blunt round in the case of the introduction of chamfering edge force, Then modified the classical cutting force model considering balance of forces of shear zone and the rake face ,more in line with the actual cutting process, accord with cutting process.

\section{Mechanical properties of precise cutting titanium alloy}

Cutting edge blunt round mechanical characteristics. Cutting speed is $100 \mathrm{~m} / \mathrm{min}$,cutting thickness for $0.04 \mathrm{~mm}$,cutting width for $0.04 \mathrm{~mm}$. Under the different cutting edge blunt round radius, numerical rules of titanium alloy cutting force in vertical direction is shown in Fig.4. 


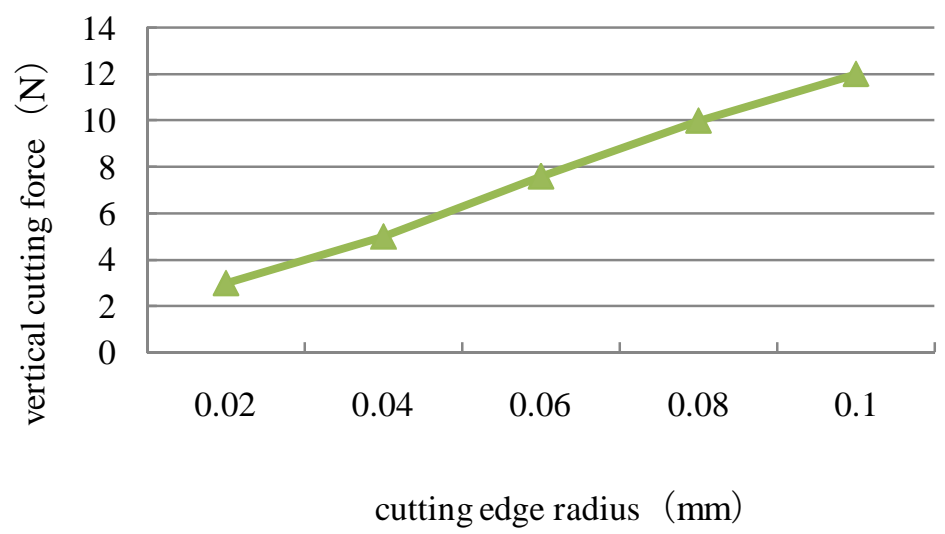

Fig.4 Simulation results of cutting force with different cutting edge radius

Through the cutting edge round radius and cutting thickness in the same order of magnitude, the cutting force numerical changes significantly. With the increase of the cutting edge blunt round radius, the cutting force increases. When cutting edge blunt round radius is $0.02 \mathrm{~mm}$, cutting force is the minimum.

Distribution state of different cutting tool cutting edge blunt round is shown in Fig.5 in finite element simulation. High stress field (red area) in the process is distributed of later cutting surface near the cutting edge. With the increase of cutting edge blunt round radius, high stress area moves to backward knife surface.

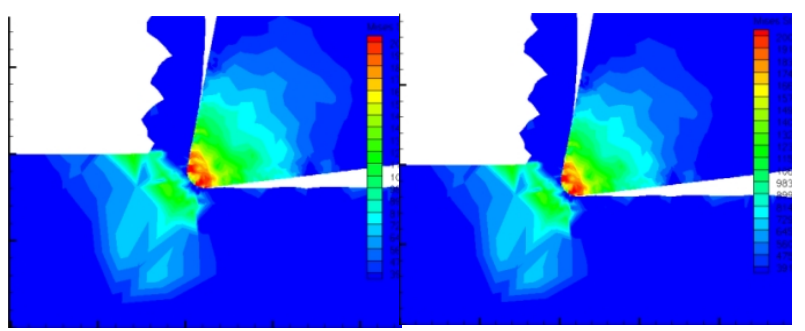
b ) $r_{n}=0.02 \mathrm{~mm}$
c ) $r_{n}=0.04 \mathrm{~mm}$

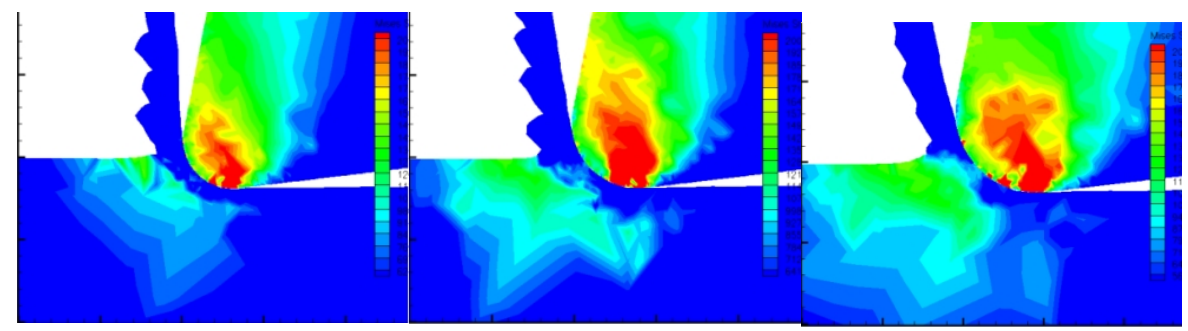
d ) $r_{n}=0.06 \mathrm{~mm}$
e ) $r_{n}=0.08 \mathrm{~mm}$
f ) $r_{n}=0.10 \mathrm{~mm}$

Fig.5 Cutting stress field distribution with different edge round radius

\section{Chamfering edge mechanical characteristics}

Using finite element analysis software, the influence rule of the vertical direction cutting force under different edge chamfer parameters is shown as fig. 6 in the same conditions of cutting layer area. Cutting way is rectangular two-dimensional cutting, cutting speed for $100 \mathrm{~m} / \mathrm{min}$, cutting thickness for $0.04 \mathrm{~mm}$, cutting width for $0.04 \mathrm{~mm}$.

It is shown inFig.6.With the increase of the edge chamfer size, the cutting force increases. The 
cutting force difference is large in different chamfer angle. The cutting force difference of same chamfer angle and different chamfering width is smaller.

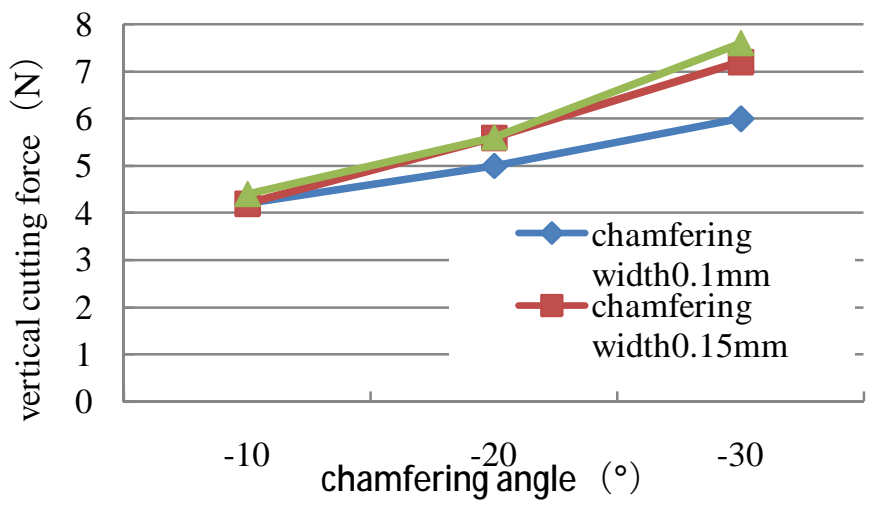

Fig.6 Variation of simulation results of cutting force with different chamfering edge

\section{Conclusions}

Cutting mechanics model is set up in cutting tool edge. Numerical analysis of cutting force is analyzed by the finite element software.Cutting mechanical characteristics analysis can provide theoretical support for tool design and $\mathrm{n}$ optimization. It can also provide effective guidance for titanium alloy membrane plate.

\section{References :}

[1] Yanyan Song, Yueqing Song. Aviation research and application progress of titanium alloy [J].

Rare Metal,2006, $12: 850-856$.

[2]Lili Han. Prediction and simulation research of the deformation of thin-walled parts processing

[D].Harbin : the dissertation of Harbin University of Science and Technology , 2009.

[3]N. Fang, Q. Wu. A comparative study of the cutting forces in high speed machining of Ti6Al4V and Inconel718 with a round cutting edge tool[J]. Journal of Materials Processing Technology, 2009, 209(9): 4385-4389 .

[4]Yihang Fan.Tool wear characteristics and cutting performance study of Efficient cutting of titanium alloy Ti6Al4V[D].Harbin : the dissertation of Harbin University of Science and Technology , 2011.

[5]Shucai Yang, Minli Zheng, Deqiang Zhang, Ning Liu and Yihang Fan. Influence of Cutting Parameters on Characteristics of Serrated Chip When High-Efficiency Cutting Ti6Al4V[C]. Solid State Phenomena, 2011, 175: 278-283. (EI: 20113214209320) 